\title{
Experimental study of water evaporation of sessile droplets on a solid substrate with different thermal conductivities
}

\author{
Vladimir Borodulin, Vladimir Letushko, Michail Nizovtsev, and Alexey Sterlyagov* \\ Kutateladze Institute of Thermophysics, 1 Ac. Lavrentiev ave., Novosibirsk, Russia
}

\begin{abstract}
The results of experimental studies of water evaporation of sessile droplet on solid substrates with different thermal conductivities are presented. In experiments during droplet evaporation the temperature of its surface was determined using the infrared thermography method. The obtained results showed that interfacial temperature was higher than the adiabatic evaporation temperature for all substrates. As thermal conductivity of the substrate decreased, the droplet temperature decreased and the evaporation lifetime increased significantly. As a result it was established that the thermal conductivity of the material has a significant effect on the evaporation of droplets.
\end{abstract}

\section{Introduction}

Evaporation of sessile droplets on solid substrates has been widely discussed in the literature due to the wide occurrence of this physical phenomenon in a variety of practical applications including formation of nanostructures [1], inkjet printing [2], fuel burning [3] and bio analyses [4]. Thus the understanding of the evaporation process of sessile droplets is important for such applications. Recently there have been many active studies of evaporation of sessile droplets on the hydrophobic textured surfaces $[5,6]$.

The process of evaporation of sessile droplets is significantly affected by the substrate material. This task is a subject of many publications in the world. Sefiane et al. $[7,8]$ showed the strong influence of the substrate conductivity on evaporation of sessile drops. In recent work Lopes et al. [9] used glass and silicon substrates with different conductivity and thermal diffusivity. They found that the thermal properties of the substrate have a strong influence on evaporation lifetime of a sessile droplet. In most of these experimental works the changing of the droplet shape was investigated, and the droplet temperature was measured using contact methods. However, the use of these methods disturbs the integrity of the drop and induces errors in results.

The aim of this paper is an experimental study of evaporating sessile drops on the substrates with different thermal conductivities. In experiments the temperature of the droplets was obtained by a non-contact method of infrared thermography. Similar experiments were previously carried out by D. Brutin et al. [10], however, in these

* Corresponding author: sterlyagov@itp.nsc.ru 
experiments infrared camera was used for visualization of thermal motion inside the evaporation sessile drop.

\section{Experimental setup}

Experimental setup was constructed in the Institute of Thermophysics to investigate sessile droplet evaporation on horozontal surfaces of substrates (see Fig.1).

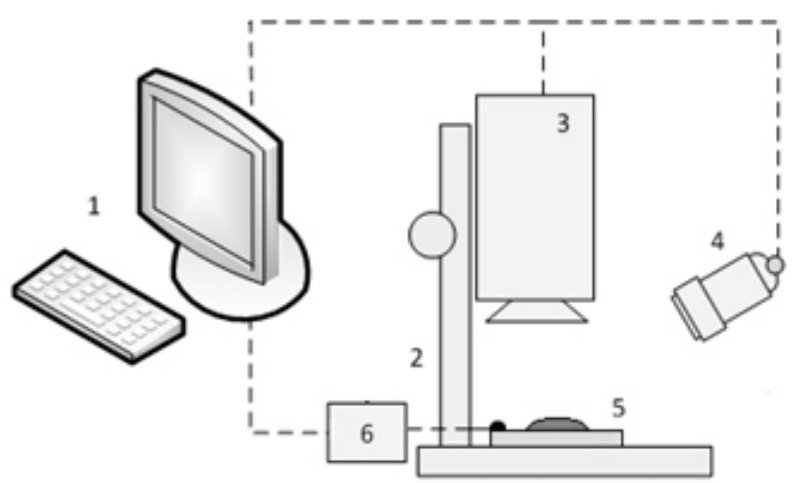

Fig. 1. Experimental setup: 1 - PC; 2 - support; 3 - infrared imaging camera; 4 - digital microscope; 5 - substrate; 6 - digital thermo-hygrometer

The infrared camera (3) was vertically located above the working area. Digital microscope (4) was located on the side of the working area. Temperature and relative humidity of ambient air were measured by thermohygrometer (6). Experimental data were collected using a computer (1).

Experiments on the evaporation of droplets were carried out as follows: The drop was first formed on the surface of substrate by mechanical pipette «Thermo Scientific» with accuracy of $\sim 0.1 \mu \mathrm{L}$. Then the local temperature distribution along the interface of an evaporating droplet was determined at different times with fixed step by infrared thermography. Infrared camera NEC TH $7102 \mathrm{WV}$ was used in experiments. It provided temperature measurements with accuracy of about $0.1{ }^{\circ} \mathrm{C}$. In order to calibrate and to determine the surface emissivity, contact measurements of the substrate temperature were additionally performed.

During the experiment, the shape of a drop of liquid was continuously recorded by a digital microscope. It allowed us to determine the change in geometric dimensions of the drop during evaporation. Initial droplet diameters were measured by digital microphotography method with an accuracy of $\pm 10 \mu \mathrm{m}$.

In experiments, the copper plates with thermal conductivity coefficient $\lambda=401 \mathrm{~W} / \mathrm{m} \cdot \mathrm{K}$, teflon plates with $\lambda=0.25 \mathrm{~W} / \mathrm{m} \cdot \mathrm{K}$ and extruded polystyrene plates (EPS) with $\lambda=0.03$ $\mathrm{W} / \mathrm{m} \cdot \mathrm{K}$ were used as substrates. The thickness of the plates was $3 \mathrm{~mm}$. A distilled water droplet with initial volume of $5 \mu \mathrm{L}$ was studied. The ambient temperature and relative humidity were set at $24{ }^{\circ} \mathrm{C}$ and $\varphi=24 \%$, respectively. At these conditions, the temperature of adiabatic evaporation or wet bulb temperature was $t_{w b}=12.7{ }^{\circ} \mathrm{C}$.

\section{Experimental results}

Interfacial temperature as function of time of water droplets sessile on the surfaces of different materials was obtained using the method of infrared thermography (Fig. 2). 


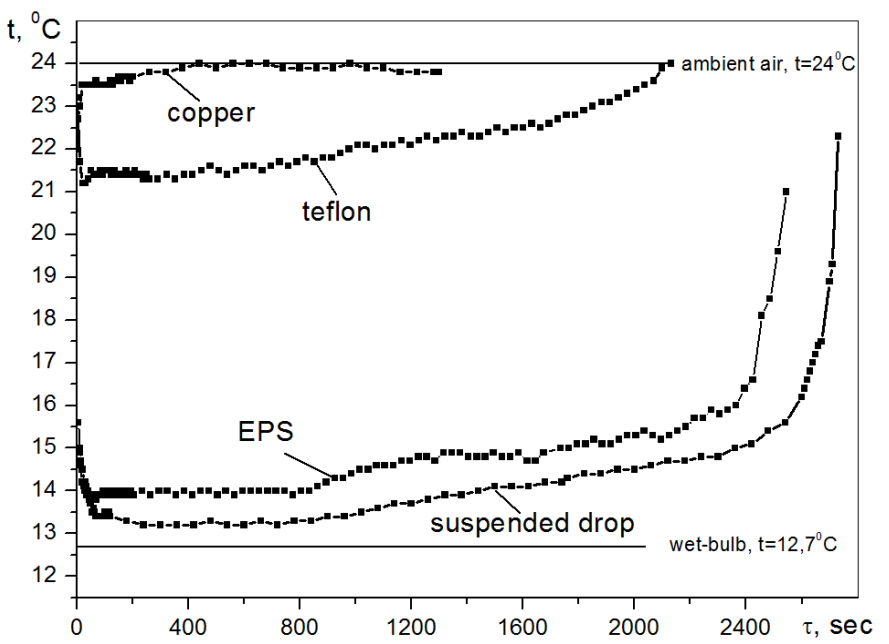

Fig. 2. Average droplet temperature on the substrate as a function of time

The behavior of temperature is seen to depend on the thermal conductivity of substrate. After initial cooling of the water drop on the copper plate, there was a fast increase of temperature up to a plate one, equal to the temperature of ambient medium. Therefore, temperature of the droplet practically did not change until the end of evaporation lifetime.

After fast cooling by $2.5^{0} \mathrm{C}$, the temperature of water droplet on the teflon substrate gradually increased to ambient temperature. Firstly droplets on the surface of EPS sharply cooled by $10.3{ }^{\circ} \mathrm{C}$. Then there was a long stage with gradually increase of temperature. At the final stage of evaporation, there was a sharp increase in temperature to temperature of ambient.

A more significant decrease of the temperature to the wet bulb temperature $t_{w b}=12.7{ }^{0} \mathrm{C}$ was observed under similar conditions for a free droplet. The presented data agree with experimental and computational data of other investigators [7, 8]. In contrast to these studies, the used method of infrared thermography allowed determining the droplet temperature without contact and obtaining data on temperature dynamics with good accuracy. As a result, the minimum droplet temperature is presented on Fig. 3 as a function of thermal conductivity of the substrate. The obtained data show that the greater the thermal conductivity of the substrate material, the smaller the minimum droplet temperature during evaporation and, respectively, the longer the evaporation time.

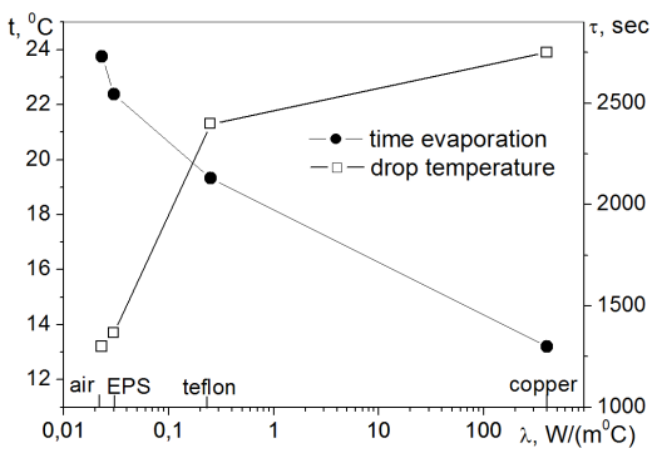

Fig. 3. Minimum temperature and evaporation lifetime of a water drop versus thermal conductivity of the substrate

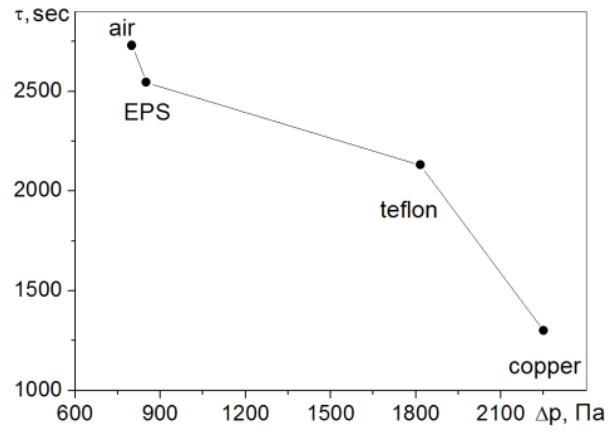

Fig. 4. Evaporation lifetime of water droplet on a substrate versus the difference in vapor pressure near the drop and in the ambient medium 
The vapor flow during droplet evaporation was determined by its diffusion into the surrounding air. The rate of evaporation depends on the difference in vapor pressures near and far from the drop surface [11]. Near the droplet surface, water vapor may be considered saturated at the surface temperature. According to the measured minimum temperatures of the droplet surfaces, the saturation pressure of the vapor near the drop was determined. The dependence of the evaporation lifetime of droplets on the difference of vapor pressure near and far from the drop is shown in Fig. 4. The obtained data show that the higher thermal conductivity of the substrate material, the greater pressure drop of vapor and, hence, the greater the intensity of evaporation.

\section{Conclusions}

As a result of experimental studies, the droplet surface temperature during evaporation was obtained for substrates with different thermal conductivity. The use of the contactless method of infrared thermography allowed obtaining data on the dynamics of temperature changes on the surfaces of evaporating droplets with high accuracy. The obtained results show that for all the considered materials, the interfacial temperature was higher than the adiabatic evaporation temperature. Moreover, the lower the thermal conductivity of the material, the lower the temperature of the drop surface. The closer this temperature to the wet bulb temperature, the longer the evaporation lifetime. The coefficient of thermal conductivity of the substrate material was found to have a significant effect on the process of evaporation of sessile droplets.

The reported study was funded by RFBR under the research project № 17-58-53168.

\section{References}

1. P.V. Lebedev-Stepanov, S.P. Molchanov, M.V. Alfimov, R.M. Kadushnikov, N.I. Rubin, N.A. Shturkin, Nanotech. 6, 79 (2011) [in Russian]

2. S.T. Beyer, K. Walus, Langmuir 28, 8753 (2012)

3. Y. Gan, L. Qiao, Int. J. Heat Mass Transfer 54, 4913 (2011)

4. D. Brutin, B. Sobac, C. Nicloux, Trans. ASME J. Heat Transfer 134, 061101 (2012)

5. Y.P. Li, M.K. Lei, J. Mater. Sci. Technol. 30, 965 (2014)

6. Z. Pan, S. Dash, J.A. Weibel, S.V. Garimella, Langmuir 29, 15831 (2013)

7. S. David, K.Sefiane, L. Tadrist, Colloids Surfaces A: Physiochem. Eng. Aspects 298108 (2007)

8. G.J. Dunn, S.K..Wilson, B.R. Duffy, S. David and K. Sefiane, J. Fluid Mech. 623, 329 (2009)

9. M.C. Lopes, E. Bonaccurso, T. Gambaryan-Roismana, P. Stephan, Colloid Surface A: Physiochem. Eng. Aspects 432, 64 (2013)

10. D. Brutin. B. Sobac, F. Rigollet, C. Le Niliot, Exp. Thermal Fluid Sci. 35, 521 (2011)

11. R. G. Picknett and R. Bexon, J. of Colloid and Interface Science 61, 336 (1977) 